\title{
Analysis of chemical consistency and the anti-tumor activity of Huangqi-Ezhu (HQ-EZ) concentrated-granules and decoction
}

\author{
Qian Wang ${ }^{1,3}$, Yongqi Dou ${ }^{2}$, Sen Zhao ${ }^{1}$, Mei Wei ${ }^{4}$, Dongmei Sun ${ }^{4}$, Youmin $\mathrm{He}^{4}$, Dequan $\mathrm{Zhu}^{4}$, Xin $\mathrm{Chen}^{5}$, \\ Heng Zhu'
}

${ }^{1}$ Chinese PLA General Hospital, Chinese PLA Medical School, Beijing, China; ${ }^{2}$ Department of Traditional Chinese Medicine, Chinese PLA General Hospital, Beijing, China; ${ }^{3}$ The Clinic of former Academy of Armored Forces Engineering, Beijng, China; ${ }^{4}$ Guangdong Yifang Pharmaceutical Co., Ltd, Foshan, China; ${ }^{5}$ Department of Burn and Plastic Surgery, Fourth Medical Center of Chinese PLA General Hospital, Beijing, China; ${ }^{6}$ Beijing Institute of Radiation Medicine, Beijing, China

Contributions: (I) Conception and design: Q Wang, Y Dou, H Zhu; (II) Administrative support: None; (III) Collection and assembly of data: S Zhao, X Chen, M Wei, D Sun, Y He, D Zhu; (IV) Provision of study materials or patients: None; (V) Collection and assembly of data: None; (IV) Data analysis and interpretation: Q Wang; (VI) Manuscript writing: All authors; (VII) Final approval of manuscript: All authors.

Correspondence to: Yongqi Dou. Department of Traditional Chinese Medicine, Chinese PLA General Hospital, Fuxing 28, Beijing, China. Email: dyqi_301@yeah.net; Heng Zhu. Beijing Institute of Radiation Medicine, Road Taiping 27, Beijing, China. Email: zhudingdingabc@163.com.

\begin{abstract}
Background: Traditional Chinese medicine decoction and modern concentrated-granules are two kinds of Chinese herbal medicine forms used in clinic at present. The former is extracted by traditional boiling method of a pre-mixed multi-herbal medicine according to the doctor's prescription. The latter is a mixture of extract active ingredients from a single variety of herbs by modern technology. It is not clear whether there is a difference in the content and efficacy of the active components between the two methods.

Methods: The effective components of Huangqi-Ezhu (HQ-EZ) traditional decoction and concentratedgranules were determined by HPLC, and the subcutaneous transplanted tumor model of tumor-bearing mice was established to compare the anti-tumor effect. HQ-EZ traditional decoction and concentrated-granules were given respectively by continuous intragastric administration for 15 days. After the last administration the tumor tissue, liver and kidney were removed completely, and the corresponding indexes were detected.

Results: Active components of concentrated-granules and traditional decoction are basically the same. Both of TCM forms have great anti-tumor effect against lung cancer, without toxify to liver and kidney.

Conclusions: The two preparation methods have their own advantages in effective components, and the compatibility of HQ-EZ can inhibit the tumor growth of tumor-bearing mice, and has no liver and kidney toxicity.
\end{abstract}

Keywords: Astragalus membranaceus; Curcuma zedoary; concentrated-granules; lung cancer

Submitted Dec 09, 2019. Accepted for publication May 25, 2020.

doi: 10.21037/apm-19-592

View this article at: http://dx.doi.org/10.21037/apm-19-592

\section{Introduction}

Decoction is the most commonly used type of traditional Chinese medicine (TCM) preparation in clinical settings. The routine steps in the preparation of decoction include soaking the plants or other components in water, boiling, removing the dregs, and extracting the juice; usually, these steps are performed by the patient at home. However, different preparation conditions, such as boiling time and cooking method, may affect the precipitation of drug components, resulting in instability of the effective components of the decoction and inconsistent quality. For a long time, thorough investigations on TCMs have been performed; these include the traditional yinpian, xiaoyinpian, boiled powder, original medicinal particles, and TCM preparations. In recent years, concentrated Chinese medicine granules have emerged as a modern form of 

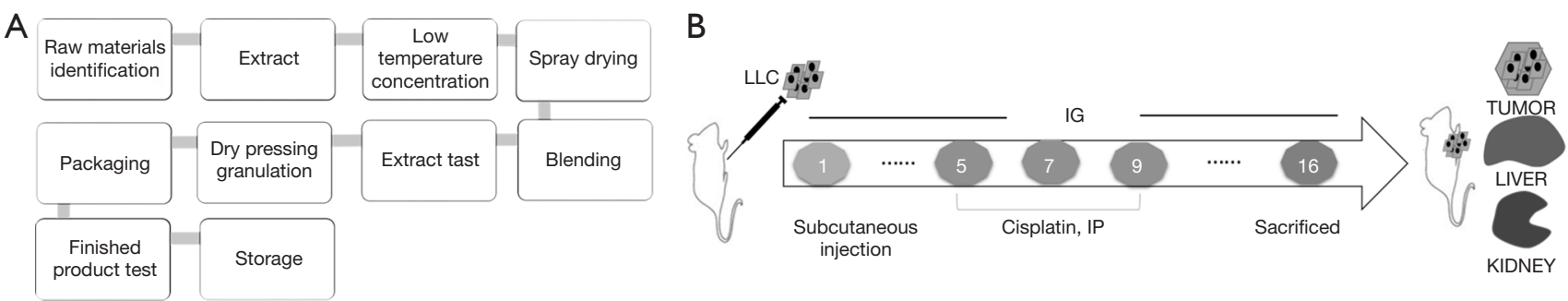

Figure 1 Preparation process of traditional Chinese Medicine concentrated granules (A) and Animal experimental process (B).

TCM, which combine modern pharmaceutical technology with the TCM decoction process, while following the basic principles of TCM health theory. The uptake of these products in clinical practice has increased.

Concentrated granules are made from good quality medicinal herbs, and utilize modern, advanced technology to manufacture products by extraction and concentration (Figure $1 A$ ). In addition, the taste and function are completely identical to original medicinal herbs. Notably, the greatest advantages of these granules are that their quality is consistent and that they are easy to consume. However, as the concentrated granules used in clinical applications are usually extracted from a single drug and then simply mixed, they lack the synergistic effects of a decoction, resulting in a considerable debate regarding the curative effect of concentrated granules, with the focus being on the differences between the co-decoction of TCM and the mixture of single-extract granules. However, supporters stress that concentrated granules have greater proportions of effective compounds, owing to their more accurate preparation methods and strict quality control during the preparation process (1); this has caused a shift in opinion, and many practitioners now believe that the quality, component extraction, and therapeutic effects of the granules are better than those prepared by traditional means.

In recent years, TCM has been used widely and effectively, with few side effects, for treating various cancers. Chinese medicine granules, such as Huaier granules (2), Renshen Yangrong Tang (3), Qingjie Fuzheng granules (4), have been used to treat different types of cancers, and can play an important role in adjuvant therapy. Further, as TCM is based on TCM theories, the adoption of syndrome differentiation treatment and TCM syndrome differentiation and typing can guide clinical trial strategies. Patients with cancer are often required to take medication for long periods of time, and there is a great demand to make medication more effective and convenient. Compared with the traditional Chinese medicine yinpian, concentrated granules are easy to take, which increases patient compliance, and offers advantages with respect to safety and hygiene (5). However, there are few comparative studies on the composition and efficacy of the two forms of TCM.

Based on our previous screening of the compatibility proportion of Huangqi-Ezhu (HQ-EZ) (6), an ancient antitumor herb pair (7), we evaluated the consistency of the pharmacological effects in the current study. The effective components of HQ-EZ concentrated granules and decoction were examined using high-performance liquid chromatography coupled with ultraviolet detection and evaporative light-scattering detection (HPLC-UVELSD), and the antitumor effects of these components were evaluated using animal experiments. It is anticipated that our study would provide scientific evidence to support the applications of concentrated TCM granules.

We present the following article in accordance with the ARRIVE reporting checklist (available at http://dx.doi. org/10.21037/apm-19-592).

\section{Methods}

\section{Plant material, chemicals and reagents}

Decoction pieces concentrate-granules of Huangqi (HQ, Astragalus membranaceus) and Ezhu (EZ, Curcuma zedoary) were provided by Guangdong Yifang Pharmaceutical Co., Ltd., and were identified as qualified medicinal materials by quality center. Astragalus membranaceus (Origin: Gansu; B/N: YL1903025), Curcuma zedoary (Origin: Guangdong; B/N: YL1905003); HQ Concentrated-granules (1 g single granules relative to $2.5 \mathrm{~g}$ slices; B/N: CG1904076), EZ Concentrated-granules $(1 \mathrm{~g}$ single granules relative to $8 \mathrm{~g}$ slices; B/N: CG1907038).

Calycosin-7-glucoside (B/N: 111920-201606; purity: 97.6\%), Formononetin (B/N: 111703-201504), Astragaloside A (B/N: 110781-201717; purity: 96.9\%) were 
purchased from China Institute of Food and Drug Control (Beijing, China); Calycosincalycosin (B/N: 20575-57-9; purity: 98\%), Astragaloside I (B/N: 84680-75-1; purity: 98\%), Astragaloside II (B/N: 84676-89-1; purity: 98\%)were purchased from shidande Standard Technical Service Co. Ltd (Shanghai, China); Curcumenol (B/N: wkq18061202; purity: 98\%) were purchased from Vicky Biotechnology Co., Ltd (Sichuan, China).

Analytical grade methanol (Xilong Science Co. Ltd, Guangdong, China, B/N: 1909021); and ethanol (Xilong Science Co., Ltd, Guangdong, China, B/N: 1909172), HPLC grade methanol (Merk, Darmstadt, Germany, B/ N:I1013907920), acetonitrile (Merk, Darmstadt, Germany, B/N: JB085430) and formic acid (Tianjin kemio Chemical Reagent Co., Ltd, Tianjin, China, B/N: 20160519) were used for the UPLC analysis. Ultra-pure water $(18 \mathrm{M} \Omega \mathrm{cm})$ was prepared in our lab by a Milli-Q water purification system (Millipore, Billerica, MA, USA).

\section{Comparison of the chemical consistency of different types of decoctions}

\section{Samples of traditional and concentrated-granule decoctions}

For the traditional decoction, the mixed herbal materials (A, $90 \mathrm{~g} ; \mathrm{C}, 30 \mathrm{~g}$ ) were accurately weighed and immersed in $1,200 \mathrm{~mL}$ water for $40 \mathrm{~min}$, and decocted by boiling for $60 \mathrm{~min}$. The solution was filtered and add $960 \mathrm{~mL}$ water into the Drug residue, boiling for $30 \mathrm{~min}$. Filtrate was concentrated to about $80-90 \mathrm{~mL}$, then transferred to $100 \mathrm{~mL}$ bottle, added water to scale with a concentration of $1.2 \mathrm{~g} / \mathrm{mL}$ (each $1 \mathrm{~mL}$ concentrated solution contains $0.9 \mathrm{~g} \mathrm{~A}$ and $0.3 \mathrm{~g} \mathrm{C}$ ). Precisely absorbed $3 \mathrm{ml}$ traditional decoction concentration, placed it in a $10 \mathrm{~mL}$ measuring bottle, added appropriate amount of methanol, treated with Ultrasonic (Power: $300 \mathrm{~W}$, frequency: $50 \mathrm{kHz}$ ) for $30 \mathrm{~min}$. After cooling, the weight loss was supplemented with $70 \%$ methanol and through a $0.22 \mu \mathrm{m}$ nylon membrane filter before analysis to obtain the traditional decoction.

For the dispensing granule decoction, samples of $\mathrm{A}$ $(1.2 \mathrm{~g}), \mathrm{C}(0.13 \mathrm{~g})$, and the mixed herbal materials (A:C=3:1, $1.33 \mathrm{~g}$ ) were accurately weighed and placed into $25 \mathrm{~mL}$ of $70 \%$ methanol, respectively for 30 min of ultrasonic treatment (Power: $300 \mathrm{~W}$, frequency: $50 \mathrm{kHz}$ ). After cooling, the weight loss was supplemented with $70 \%$ methanol and through a $0.22 \mu \mathrm{m}$ nylon membrane filter before analysis to obtain dispensing granule decoctions.

\section{Solutions of control sample}

Accurately weighed Calycosin $2.092 \mathrm{mg}$, Calycosin-7glucoside $3.090 \mathrm{mg}$, Formononetin $2.064 \mathrm{mg}$, Curcumenol $2.103 \mathrm{mg}$. Dissolved with methanol and diluted to $10 \mathrm{~mL}$, shook well to obtain the mixed control sample of astragalus flavonoids and Curcumenol (I). Accurately weighed Astragaloside A $2.136 \mathrm{mg}$, Astragaloside I $2.008 \mathrm{mg}$, Astragaloside II $2.044 \mathrm{mg}$. Dissolved with methanol and diluted to $20 \mathrm{~mL}$, shook well to obtain the mixed control sample of Astragalus saponins (II).

\section{Chromatographic conditions optimization}

An AcclaimTM 120 C18 column $(250 \mathrm{~mm} \times 4.6 \mathrm{~mm}, 5 \mu \mathrm{m})$ was applied for all the analysis. The mobile phase consisted of A (acetonitrile) and B ( $0.5 \%$ formic acid) using a gradient elution, which programmed as follows: $0-9 \mathrm{~min}, 20-25 \% \mathrm{~A}$; 9-24 min, 25-32\% A; 24-29 min, 32-43\% A; 29-37 min, $43-46 \%$ A; $37-47 \mathrm{~min}, 46-90 \% \mathrm{~A}$. The flow rate was set at $1.0 \mathrm{~mL} / \mathrm{min}$ with $10 \mu \mathrm{L}$ per injection. Detection wavelength was $254 \mathrm{~nm}$. The column and autosampler temperature were conditioned at $35^{\circ} \mathrm{C}$. The drift tube temperature of evaporative light scattering detector is $101{ }^{\circ} \mathrm{C}$ and the carrier velocity is $2.5 \mathrm{~mL} / \mathrm{min}$.

\section{Calibration curves}

The standard solution was diluted to prepare a series of appropriate concentrations for the establishment of calibration curves. Precision absorbed the mixed reference solution of astragalus flavonoids and Curcumenol (I) $0.5,1.0,1.0,2.0 \mathrm{~mL}$ in $20,20,10,5,5 \mathrm{~mL}$ measuring bottles, diluted with methanol to scale, fully shake well. According to the above chromatographic conditions, the chromatographic peak area was determined by injection in turn, and the chromatographic peak area was recorded. The standard curve was drawn with the peak area as the longitudinal coordinate (y), reference concentration as the Abscissa (x), the results were shown in Table 1. The four chemical components of astragalus flavonoids and Curcumenol had a good relationship in a certain linear range ( $r>0.9995)$, and had high sensitivity.

Precision absorbed the mixed reference solution of Astragalus saponins (II) 1.0, 1.0, 1.0, $2.0 \mathrm{~mL}$ in 20, 20, 10, $5,5 \mathrm{~mL}$ measuring bottles, diluted with methanol to scale, fully shake well. According to the above chromatographic conditions, the chromatographic peak area was determined 
Table 1 Linear regression equation of each component

\begin{tabular}{lcccc}
\hline Control sample & $\mathrm{t}(\mathrm{min})$ & Regression equation & $\mathrm{r}$ value & Linear range $(\mu \mathrm{g})$ \\
\hline Calycosin-7-glucoside & 7.45 & $\mathrm{y}=2.78 \times 10^{7} \mathrm{x}+1.82 \times 10^{4}$ & 0.9998 & $7.5396-301.5840$ \\
Calycosin & 21.62 & $\mathrm{y}=4.89 \times 10^{7} \mathrm{x}+1.06 \times 10^{4}$ & 1.0000 & $5.1254-205.0160$ \\
Formononetin & 33.86 & $\mathrm{y}=5.92 \times 10^{7} \mathrm{x}+1.66 \times 10^{4}$ & 1.0000 & $5.1600-206.4000$ \\
Curcumenol & 43.10 & $\mathrm{y}=1.35 \times 10^{7} \mathrm{x}-1.27 \times 10^{3}$ & 1.0000 & $5.1524-206.0940$ \\
Astragaloside A & 33.40 & $\mathrm{y}=1.77 \mathrm{x}+6.76$ & 0.9997 & $5.1745-103.4892$ \\
Astragaloside I & 36.53 & $\mathrm{y}=1.72 \mathrm{x}+6.61$ & 0.9994 & $4.9196-98.3920$ \\
Astragaloside II & 43.30 & $\mathrm{y}=1.77 \mathrm{x}+6.71$ & 1.0000 & $5.0078-100.1560$ \\
\hline
\end{tabular}

by injection in turn, and the chromatographic peak area was recorded. The standard curve was drawn with the peak area as the longitudinal coordinate (y), reference concentration as the Abscissa (x), the results were shown in Table 1. The three chemical components of Astragalus saponins had a good relationship in a certain linear range ( $r>0.9990)$, and had high sensitivity.

\section{Precision inspection}

Precision absorbed the mixed reference solution of astragalus flavonoids and Curcumenol (I) and the mixed reference solution of Astragalus saponins (II), repeated 6 times according to the above chromatographic conditions, recorded the chromatographic peak area, and calculated the relative standard deviation (RSD) of the peak area. The result showed that, the peak area RSD of Calycosin7-glucoside, Calycosin, Formononetin, Curcumenol, Astragaloside A, Astragaloside I, Astragaloside II were $0.92 \%, 1.21 \%, 0.84 \%, 0.58 \%, 1.38 \%, 1.15 \%, 0.90 \%$, respectively, showed that the instrument was accurate and good.

\section{Repeatability inspection}

Accurately weighed six samples of the mixed herbal materials (A:C=3:1, $1.33 \mathrm{~g}$ ), and prepared six solutions according to the methods of " 1.1 ". Then measured the RSD of effective components according to the above chromatographic conditions, and calculated the RSD of Calycosin-7-glucoside, Calycosin, Formononetin, Curcumenol, Astragaloside A, Astragaloside I, Astragaloside II. Results were $1.02 \%, 0.28 \%, 2.12 \%, 0.85 \%, 2.12 \%$, $0.57 \%, 1.01 \%$, s proved that experimental method repeatability was good.

\section{Stability inspection}

Prepared the sample solution for stability inspection, and injected at $0,2,4,8,12,24$ hours according to the above chromatographic conditions. Peak areas RSD of Calycosin7 -glucoside, Calycosin, Formononetin, Curcumenol, Astragaloside A, Astragaloside I, Astragaloside II were $0.29 \%, 0.86 \%, 1.53 \%, 1.33 \%, 1.06 \%, 0.85 \%, 1.93 \%$, proved that the solution was stable within 24 hours.

\section{Recovery test}

Accurately weighed the mixture of A and C (3:1), and paralleled in 6 parts. Added the reference substance with the same content as each component in the sample, and prepared the sample solution according to the method of "1.1". Detected the sample and record the peak area of each component using the same chromatographic conditions, and calculated the recovery rate and RSD value. The result showed recovery rates of Calycosin-7-glucoside, Calycosin, Formononetin, Curcumenol, Astragaloside A, Astragaloside I, Astragaloside II were 95.4\%, 92.8\%, 91.2\%, 98.6\%, $100.6 \%, 93.5 \%, 101.6 \%$, and $\mathrm{RSD}$ value are $2.01 \%, 1.43 \%$, $1.72 \%, 2.05 \%, 2.36 \%, 1.57 \%, 1.85 \%$, proved that the accuracy of this method was good.

\section{Comparison of the in vivo anti-tumor activity}

Lewis lung carcinoma (LLC), a mouse lung cancer cell line, were obtained from the National Infrastructure of Cell Line Resource (Beijing, China). The cells were cultured in Dulbecco's Modified Eagle's Medium (DMEM; Hyclone, Logan, UT, USA), with $10 \%$ fetal bovine serum (FBS; Hyclone), and were cultured in a cell incubator supplied with $5 \% \mathrm{CO}_{2}$ at $37^{\circ} \mathrm{C}$.

Male C57BL/6 mice [18-20 g, production certificate: 
SCXK (Jing 2016-0001)] were obtained from Beijing Vital River Laboratory Animal Technology Co., Ltd (Beijing, China). All animals received humane care according to the National Institutes of Health guidelines were housed in standardized conditions in animal facilities at $22 \pm 2{ }^{\circ} \mathrm{C}$ room temperature, $55 \% \pm 5 \%$ relative humidity and a $12 \mathrm{~h} \mathrm{light/}$ dark cycle with dawn/dusk effect in the animal center of Institute of military Medicine (Beijing, China). Water and standard pathogen-free chow diet were provided ad libitum.

For the subcutaneous xenograft model, LLC cells $\left(0.1 \mathrm{~mL}, 1 \times 10^{7}\right)$ were injected subcutaneously into the right forearm axilla of mice. On the second day, the 40 mice were randomly divided into four groups: Group 1 (negative control), Group 2 (positive control), Group 3 (traditional decoction), and Group 4 (concentrated granules). The animals were given normal saline (NS) (group 1 and 2, $0.2 \mathrm{~mL}$ ) or the respective Chinese medicine decoction liquid (group 3 and $4,11 \mathrm{~g} / \mathrm{kg}$ ) by gavage once every morning for 15 days. In group 2, mice were intraperitoneally injected with Cisplatin (Qilu Pharmaceutical, Hainan, China, B/N: AA1A7030B) prepared with normal saline at $5 \mathrm{mg} / \mathrm{kg}$ on days 5,7 , and 9 after the subcutaneous injection of LLC tumor cells (Figure 1B).

\section{General condition and tumor inhibition rate}

We observed the mental state, activity, hair glossiness, and stool character of mice in each group every day. To monitor the toxicity of the extracts, the body weight of each animal was measured every 2 days by an electronic scale. After the tumor was palpable (about 5 days after inoculation), we measured the length and width every two days with an electronic Vernier caliper. The tumor volume in mice was measured and calculated according to the formula $1 / 2 \times$ length $\times$ width $^{2}$. Mice were sacrificed 24 hours after the last intragastric administration. The tumor tissue was stripped and weighed, and the tumor index (tumor weight index $=$ tumor weight/body weight) and tumor inhibition rate $\{$ tumor inhibition rate $=[$ (average tumor weight in the model group - average tumor weight in the administration group)/average tumor weight in the model group] $\times 100 \%$ \} were calculated.

\section{HE immunohistochemistry on formalin-fixed paraffin-embedded (FFPE) samples}

The xenograft tumor tissues, kidneys, and livers were harvested and washed with NS, then fixed in $10 \%$ neutralbuffered formalin, then dehydrated by immersion in a series of ethanol dilutions in water $(80 \%, 90 \%$, and $95 \%)$ and followed by immersion in absolute ethanol. Afterward, they were embedded in paraffin blocks and cut into $4 \mu \mathrm{m}$ thick tissue sections. The presence of tumor was confirmed on hematoxylin \& eosin (H\&E) staining. The tumor cell morphology of each group was observed by K-Viewer 1.5.3.1 software.

\section{Statistical analysis}

Data were analyzed in GraphPad Prism 6 software. Pairwise comparisons were performed using an unpaired Student's $t$-test. Grouped data were assessed by two-way ANOVA with Bonferroni's correction to adjust for multiple comparisons. Tumor volumes were analyzed by twoway ANOVA followed by Sidak correction for multiple comparisons. Statistical details can be found in the figure legends.

\section{Results}

\section{Active components in concentrated granules and traditional decoction are essentially the same}

In our study, the described UPLC method was applied for the quantitative analysis of compounds in different types of decoctions. The results of the quantitative analysis indicated that the number and type of chemical compounds were similar between concentrated granules and traditional decoction (Figure 2). Further data confirmed those compounds by comparison with standard compounds, showing that the chemical concentrations had only minor differences (Table 2). For example, the content of calycosin7-glucoside and Astragaloside A in HQ-EZ concentrated granules was higher than that in traditional decoction, but the calycosin, formononetin, curcumenol, Astragaloside I, and Astragaloside II content in the HQ-EZ traditional decoction were higher than those in concentrated granules. Detailed information about the compounds identified was listed in Figure 3.

\section{HQ-EZ can improve overall bealth in the mouse tumor model}

The present study investigated antitumor effects on subcutaneous transplanted tumors of LLC cells in mice. The appearance of tumors was shown in Figure $4 \mathrm{~A}$. Compared with group 2, the mental state, hair glossiness, 

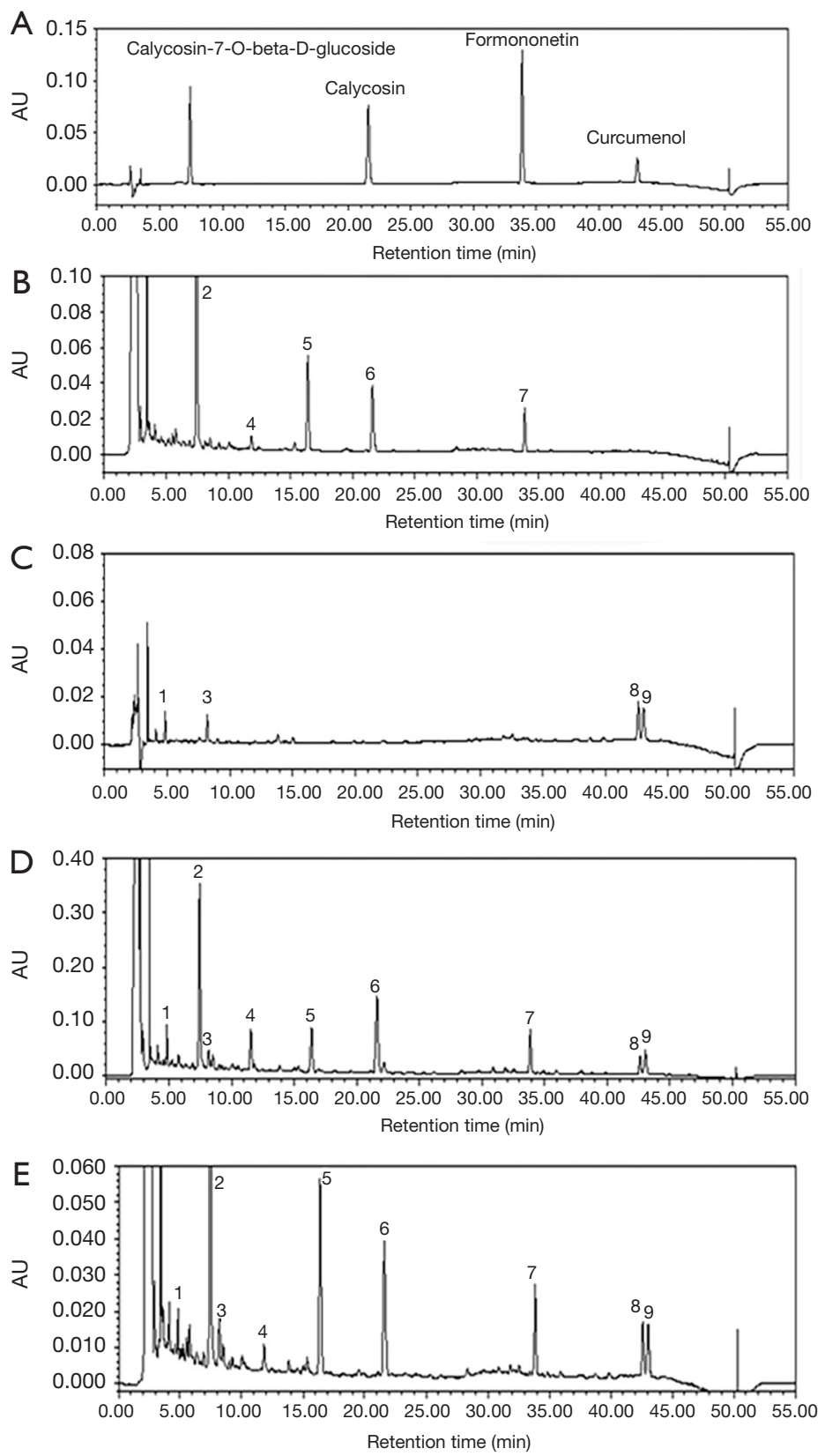

Figure 2 HPLC fingerprints spectrum (UV) of standard sample (A) and identification of common peaks in Hungqi (HQ) (B), Ezhu (EZ) (C), HQ-EZ traditional decoction (D), and HQ-EZ concentrated granules (E).

weight gain (Figure 4B), activity, and response to external stimuli in TCM groups were better, and the growth rate of the axillary tumor was much slower (Figure $4 C$ ). No significant differences were observed between the traditional decoction group and the concentrated granule group.

\section{Concentrated granules and traditional decoction both can inhibit tumor growth}

Compared with the model group (group 1), the positive control group showed that treatment with dexamethasone cisplatin $(5 \mathrm{mg} / \mathrm{kg})$ can significantly inhibit tumor growth 
Table 2 Determination of active components in different samples $(\mathrm{mg} / \mathrm{g})$

\begin{tabular}{|c|c|c|c|c|c|c|c|}
\hline Test sample & Calycosin-7-glucoside & Calycosin & Formononetin & Curcumenol & Astragaloside A & Astragaloside I & Astragaloside II \\
\hline EZ granules & - & - & - & 0.295 & - & - & - \\
\hline $\begin{array}{l}\text { HQ-EZ concentrated } \\
\text { granules }\end{array}$ & 0.477 & 0.079 & 0.032 & 0.291 & 0.148 & 0.184 & 0.097 \\
\hline
\end{tabular}

"-" indicates that the value is below the detection limit. The content of each component was relative to the content per $g$ of Astragalus membranaceus and Curcuma zedoary.

A

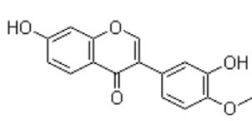

Calycosin MF: $\mathrm{C}_{17} \mathrm{H}_{14} \mathrm{O}_{5}$ MW: 284.27
B

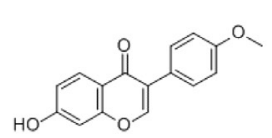

Formononetin MF: $\mathrm{C}_{16} \mathrm{H}_{12} \mathrm{O}_{4}$ MW: 268.27
C

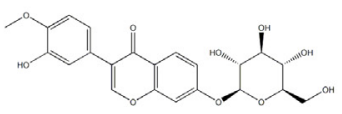

Calycosin-7-O-beta-D-glucoside MF: $\mathrm{C}_{22} \mathrm{H}_{22} \mathrm{O}_{10}$ MW: 446.404

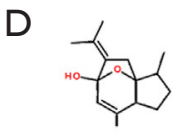

Curcumenol MF: $\mathrm{C}_{15} \mathrm{H}_{22} \mathrm{O}_{2}$ MW: 234.34

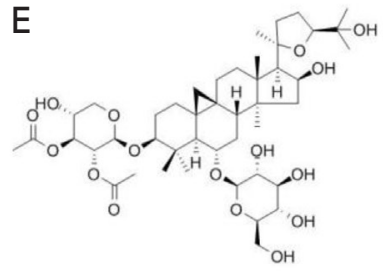

Astragaloside I MF: $\mathrm{C}_{45} \mathrm{H}_{72} \mathrm{O}_{16}$ MW: 869.07

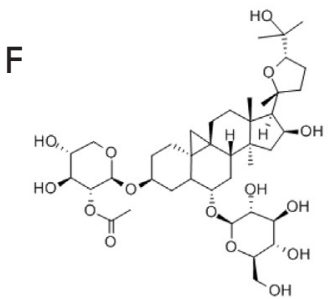

Astragaloside II MF: $\mathrm{C}_{43} \mathrm{H}_{70} \mathrm{O}_{15}$ MW: 827.03

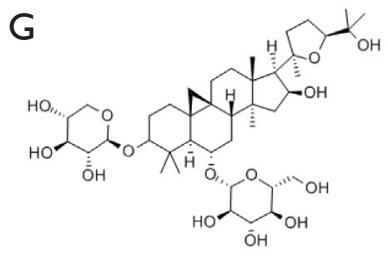

Astragaloside A MF: $\mathrm{C}_{41} \mathrm{H}_{68} \mathrm{O}_{14}$ MW: 784.974

Figure 3 Chemical structures of seven active ingredients.

in mice. Compared with the model group, a significant difference was also observed in groups 3 and $4(\mathrm{P}<0.01)$, whereas no significant difference was observed between those two groups $(\mathrm{P}>0.05)$. The results showed that both HQ-EZ concentrated granules and traditional decoction could significantly decrease percent tumor growth inhibition (Figure $5 A, B$ ). Comparing the two groups, the antitumor effect of concentrated granules was better, and the difference was statistically significant $(\mathrm{P}<0.05)$.

\section{Tumor histopatbology}

The largest cross section of mouse tumor tissue collected was selected for HE staining. Results (Figure 5C) showed the tumor cells in each group were arranged densely and the morphology was diverse. Compared with the three treatment groups, in the model group, the proportion of nucleus to cytoplasm was increased, nuclear pyknosis was decreased, the necrotic area was smaller, and the number of pathological mitotic cells was greater. However, the necrotic area in the cisplatin group was the largest, and no significant difference was observed between the two TCM groups.

\section{Neither concentrated granules nor traditional decoction show toxicity to the liver and kidneys}

To evaluate the safety of concentrated granules and traditional decoction, histopathological analysis of liver samples was conducted, and the results (Figure 6 $A$ ) revealed that the tissue sections had no obvious abnormalities in 
A

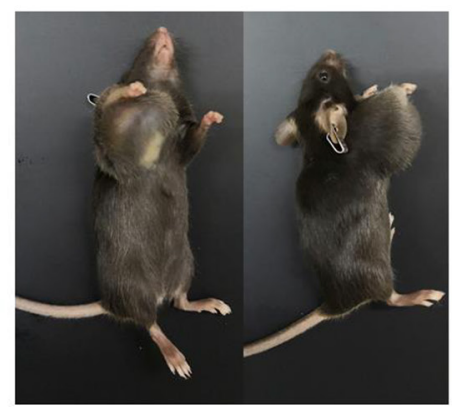

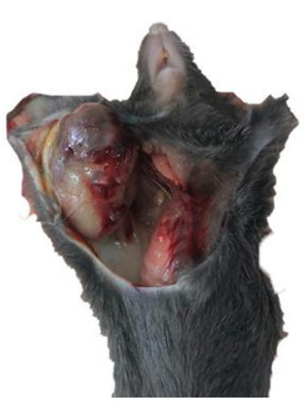

B

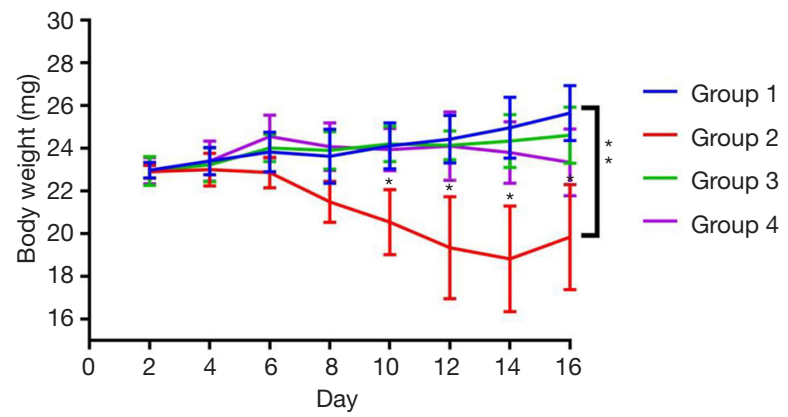

C

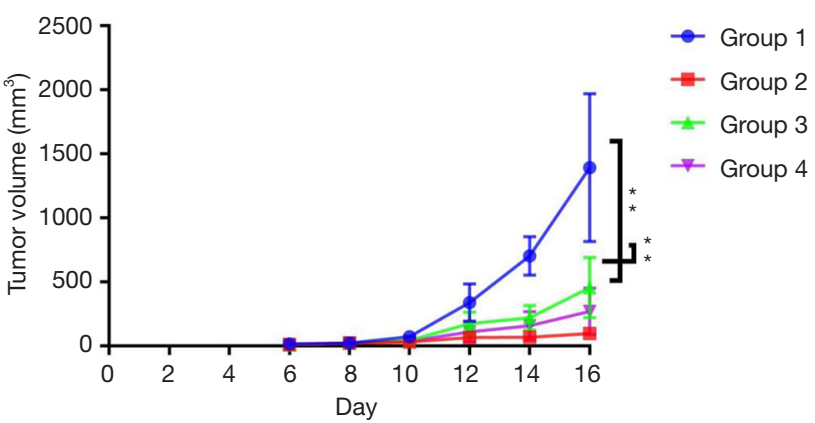

Figure 4 Appearance of mice after Subcutaneous transplantation of tumor (A). Body weight curve (B) and Volume changes of subcutaneous transplanted tumor $(C)$ of mice. Each value represents mean $\pm \mathrm{SD}$. ${ }^{*} \mathrm{P}<0.05$ and ${ }^{* *} \mathrm{P}<0.01$ compared with the concentration of group 1.

their gross anatomy. Histopathological examination showed normal hepatic lobules with granulated and radiating hepatocytic cords from the central vein, the cells were regular and stained evenly, no pigment granules had been deposited, and no obvious histopathological changes were observed. Furthermore, results (Figure 6B) of renal histopathological examination showed that in the cisplatin group, there was a small amount of capillary congestion in the renal cortex and glomeruli, red blood cells in the lumen, and necrosis and apoptosis of renal tubular epithelial cells. Kidney samples from the control group and the two TCM groups showed normal glomerular morphology, with no congestion in capillaries, and with tight arrangement of renal tubules and regular arrangement of renal tubular epithelial cells. The morphology was normal, and there were no obvious histopathological changes.

\section{Discussion}

Cancer is a major public health concern worldwide (8). It is the leading cause of death and the single most important bottleneck to increasing the global life expectancy (9).
Currently, surgery, radiation therapy, and systemic therapies, which include chemotherapy, targeted therapy, and immunotherapy, are the three most common treatment modalities used for patients with malignant tumors (10). However, many challenges remain, including the severe adverse effects caused by chemotherapy and radiotherapy, and the development of drug resistance over time. In China, herbal medicine is frequently combined with other treatments to reduce adverse effects $(11,12)$, improve patient quality-of-life, and prolong survival. Many natural products that originate from Chinese herbal medicine exhibit anticancer activities (13-15), including antiproliferative, pro-apoptotic, antimetastatic, and anti-angiogenic effects. These products have been shown to regulate autophagy, reverse multidrug resistance, balance immunity, and enhance chemotherapy in vitro and in vivo (16).

Decoction is one of the classic TCM preparations. The type and dosage of prepared components in TCM can be adjusted based on syndrome differentiation and treatment (17); indeed, this is the essence of TCM practice. However, the sources and the material bases of TCM are diverse, as are the preparation techniques. The quality 
A

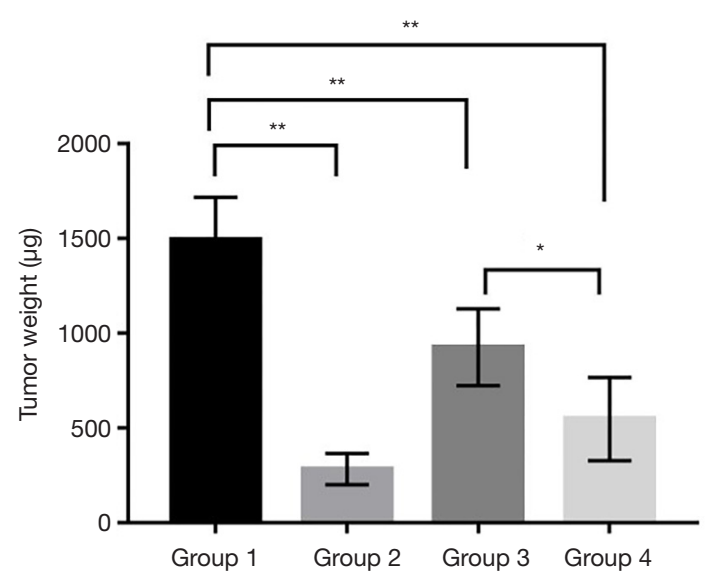

B

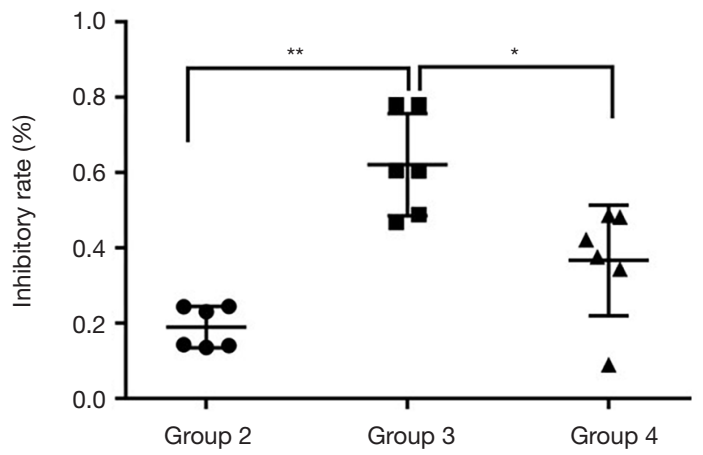

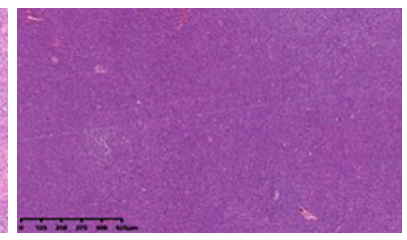
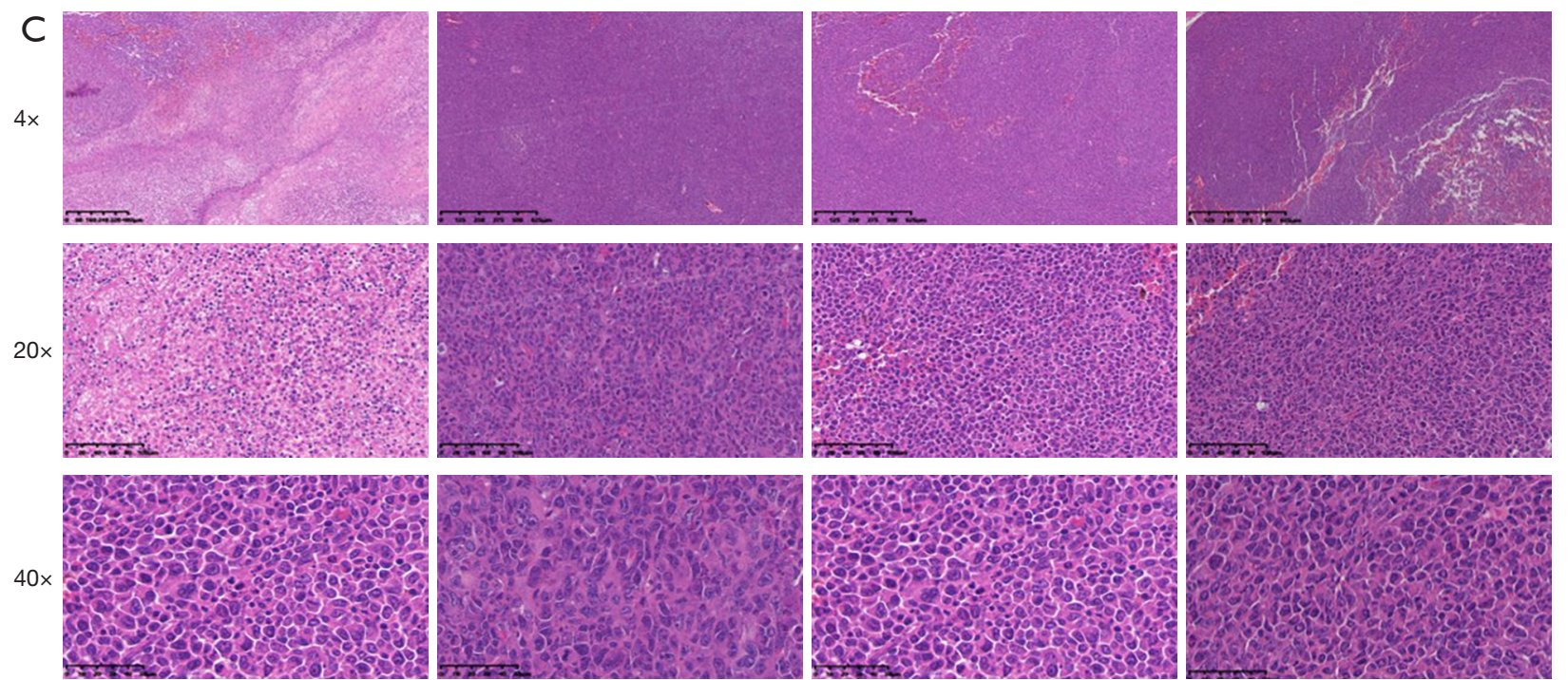

Group 1

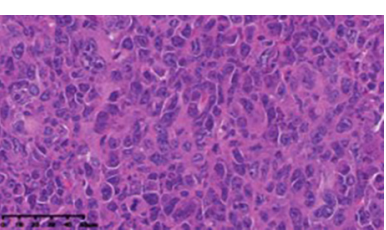

Group 2
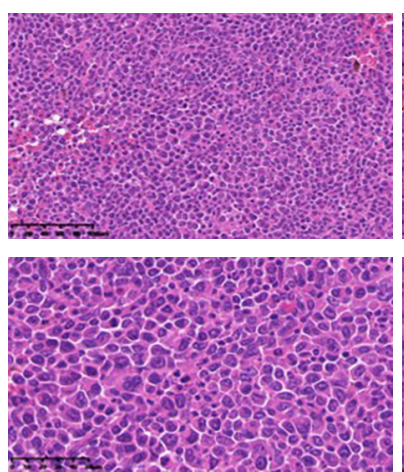

Group 3
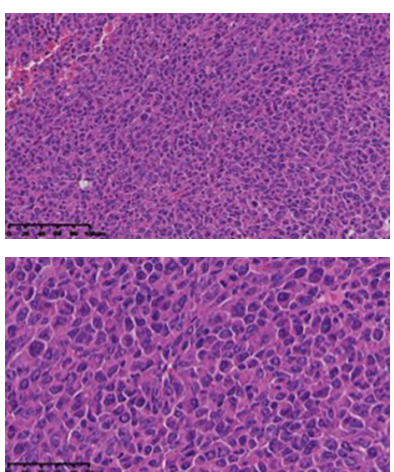

Group 4

Figure 5 Tumor weight (A) and inhibition rate of three treatment group (B) were shown. * and ** indicate significant differences relative to group 1 at $\mathrm{P}<0.05$ and $\mathrm{P}<0.01$. (C) Hematoxylin-eosin staining images. Black bars indicate 400, 100 and $50 \mu \mathrm{m}$ of magnification.

control of TCM decoctions is complex, and reliable quality control standards are lacking. Concentrated granules are able to compensate for the shortcomings of decoctions. There is no need to cook them; they are easy to preserve, carry, and consume; and they are stable. Therefore, the determination of the similarities and differences between the traditional decoction and the concentrated granules with respect to the effective components and curative effects is clinically significant.

In the present study, we used four sample solutions to compare the active components produced upon using different preparation methods. HQ concentrated granules-extracted from the dried root of Astragalus membranaceus (Fisch.) Bge. var. mongholicus (Bge.) Hsiao or A. membranaceus (Fisch.) Bge (18)—mainly contain astragaloside A (19), calycosin-7-glucoside (20), and astragalus polysaccharides (21); among the active compounds present in HQ concentrated granules, astragalus saponins and flavonoids are the main compounds with antitumor activity. EZ concentrated granules-extracted from the dried root of Curcuma phaeocaulis Val, Curcuma kwangsiensis S. G. Lee et C. F. Liang, and Curcuma wenyujin Y. H. Chen et C. Ling-mainly contain volatile oils (22); curcumin (23), elemene (24), curcumol (25), and curcumone were the main antitumor compounds in EZ concentrated granules. We used HPLC-UV-ELSD to identify the active compounds 


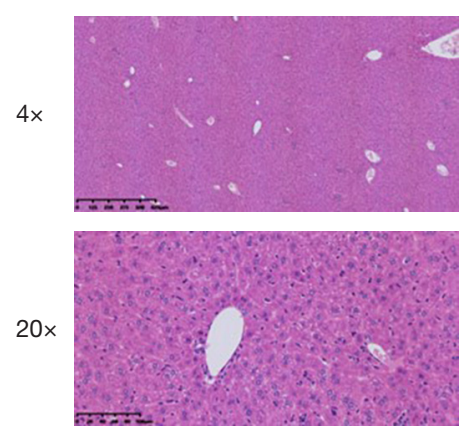

Group 1

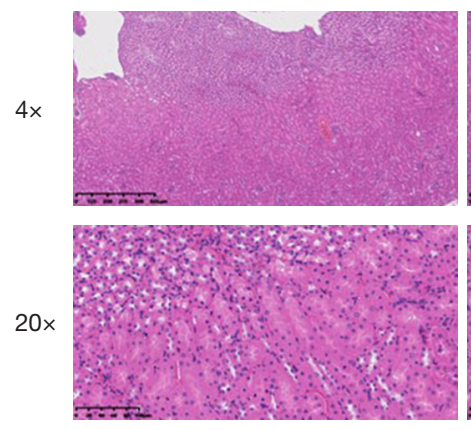

Group 1
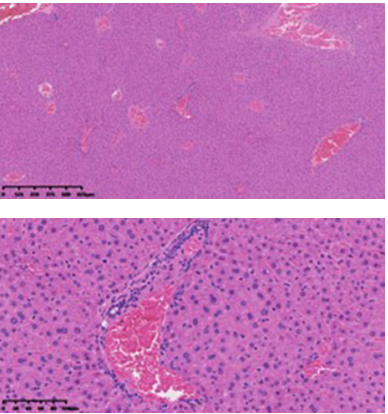

Group 2

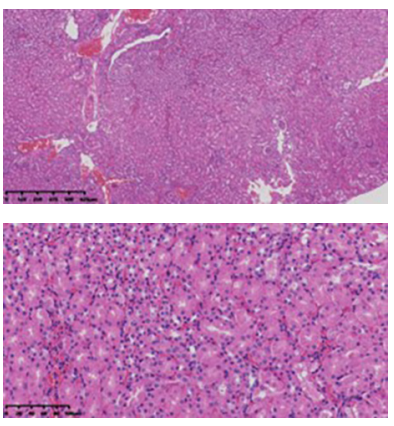

Group 2
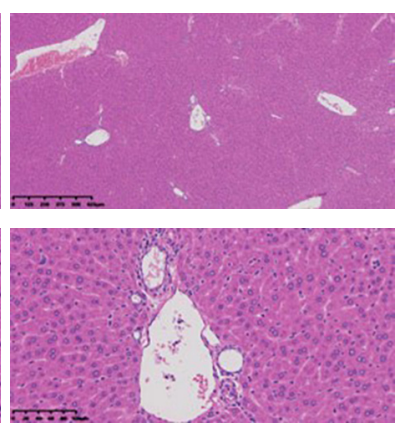

Group 3

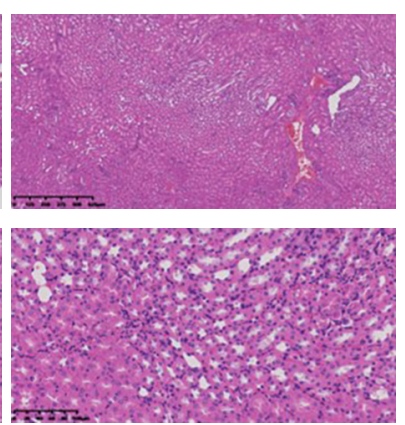

Group 3

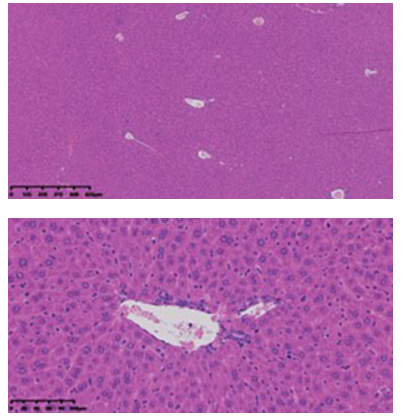

Group 4

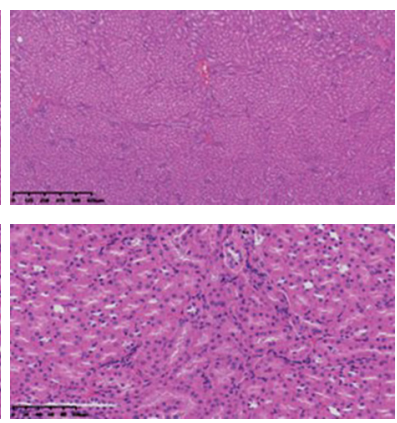

Group 4

Figure 6 Histopathological analysis of hepatic sections (A) and kidney (B) stained with H\&E.

in HQ-EZ after preparation using the two methods, i.e., decoction preparation and concentrated granule preparation, and the results showed that the types of active compounds were essentially the same. Subsequently, we calculated the RSD value for each sample and found that there were some differences between the two preparation methods. We showed that the extraction efficiency of concentrated granules was higher for calycosin-7-glucoside and astragaloside A. The decoction mixture of $\mathrm{HQ}$ and $\mathrm{EZ}$ enabled the precipitation of calycosin, formononetin, curcumenol, astragaloside I, and astragaloside II to a greater level than the concentrated granule preparation, which confirmed that the process of decoction resulted in a unique phenomenon with advantages such as solubilization of the components, which arise from the complex physical and chemical interactions between the components of different drugs (26). The two preparation methods have their own advantages.

Clinical efficacy is the most important criterion when testing the effects of drugs. For a further comparison of the similarities and differences between the two different preparations, we designed an in vivo experiment to observe the antitumor effects and stability. Based on previous experimental studies, we used the model of subcutaneous tumorigenesis in tumor-bearing mice to investigate the effects of these preparations. The anticancer drug cisplatin was used as a positive control, and its effects were compared with those of TCM decoction prepared using different methods and administered via the intragastric route. The results showed that the tumor inhibition rate of the concentrated granules was $63.3 \%$, and that its curative effect was better than that of the traditional mixture. To evaluate the safety of the concentrated granules, we looked for pathological changes in the liver and kidney tissues of mice. The results showed that the products were safe for oral administration and exhibited no adverse effects on liver or kidney function.

A limitation of this study is that we did not identify and compare any other, less abundant components present in the concentrated granules and decoctions, and we did not confirm whether the different components contributed to the anticancer effect.

\section{Conclusions}

In this study, a comprehensive analysis of chemical composition and the antitumor activity of HQ-EZ decoction and concentrated granules was performed. Our 
initial results showed that the two preparation methods have their own advantages, and that HQ-EZ could inhibit tumor growth in tumor-bearing mice, without inducing liver or kidney toxicity.

\section{Acknowledgments}

Funding: This work received financial support from National Natural Science Foundation of China (No. 81673810).

\section{Footnote}

Reporting Checklist: The authors have completed the ARRIVE reporting checklist. Available at http://dx.doi. org/10.21037/apm-19-592

Data Sharing Statement: Available at http://dx.doi. org/10.21037/apm-19-592

Conflicts of Interest: All authors have completed the ICMJE uniform disclosure form (available at http://dx.doi. org/10.21037/apm-19-592). The authors have no conflicts of interest to declare.

Ethical Statement: The authors are accountable for all aspects of the work in ensuring that questions related to the accuracy or integrity of any part of the work are appropriately investigated and resolved. Experiments were performed under a project license granted by the Institutional Animal Care and Use Committee of Chinese PLA general hospital, in compliance with the law and regulation stipulation for the care and use of animals.

Open Access Statement: This is an Open Access article distributed in accordance with the Creative Commons Attribution-NonCommercial-NoDerivs 4.0 International License (CC BY-NC-ND 4.0), which permits the noncommercial replication and distribution of the article with the strict proviso that no changes or edits are made and the original work is properly cited (including links to both the formal publication through the relevant DOI and the license). See: https://creativecommons.org/licenses/by-nc-nd/4.0/.

\section{References}

1. Zhen-Yun H, Shu-Ting C, Fei-Shu Z, et al. Research and analysis about the problems of Chinese medicinal granules in clinical application. China Journal of Traditional Chinese Medicine and Pharmacy 2017;32:2862-4.

2. Wang M, Hu Y, Hou L, et al. A clinical study on the use of Huaier granules in post-surgical treatment of triplenegative breast cancer. Gland Surg 2019;8:758-65.

3. Xu Y, Wang XS, Chen Y, et al. A Phase II Randomized Controlled Trial of Renshen Yangrong Tang Herbal Extract Granules for Fatigue Reduction in Cancer Survivors. J Pain Symptom Manage 2020;59:966-73.

4. Yang H, Liu JX, Shang HX, et al. Qingjie Fuzheng granules inhibit colorectal cancer cell growth by the PI3K/ AKT and ERK pathways. World J Gastrointest Oncol 2019;11:377-92.

5. Zhang C, Chen S, Li B, et al. HPLC fingerprint of Cnidii Fructus formula granule and determination of six main coumarin components. Chinese Traditional and Herbal Drugs 2020;51:1530-6.

6. Xu C, Xu R, Wang Y, et al. Inhibitive effects and mechanism of the compatibility of astragali radix and curcumae rhizome on the growth, metastasis and angiogenesis in Lewis lung carcinoma. World Journal of Integrated Traditional and Western Medicine 2018;13:596-8.

7. Tang D, Zhang S, Shi X, et al. Combination of Astragali Polysaccharide and Curcumin Improves the Morphological Structure of Tumor Vessels and Induces Tumor Vascular Normalization to Inhibit the Growth of Hepatocellular Carcinoma. Integr Cancer Ther 2019;18:1534735418824408.

8. Siegel, Rebecca L, Miller, Kimberly D, Jemal, Ahmedin. Cancer statistics, 2019. CA Cancer J Clin 2019;69:7-34.

9. Bray F, Ferlay J, Soerjomataram I, et al. Global cancer statistics 2018: GLOBOCAN estimates of incidence and mortality worldwide for 36 cancers in 185 countries. CA Cancer J Clin 2018;68:394-424.

10. Wood DE, Kazerooni EA, Baum SL, et al. Lung Cancer Screening, Version 3.2018, NCCN Clinical Practice Guidelines in Oncology. J Natl Compr Canc Netw 2018;16:412-41.

11. Yang $Y$, Sun $M$, Yao W, et al. Compound kushen injection relieves tumor-associated macrophage-mediated immunosuppression through TNFR1 and sensitizes hepatocellular carcinoma to sorafenib. J Immunother Cancer 2020;8:e00317.

12. Lin WF, Lu JY, Cheng B, et al. Progress in research on the effects of traditional Chinese medicine on the tumor microenvironment. Journal of Integrative Medicine 2017;15:282-7. 
13. Ye J, Zhang R, Wu F, et al. Non-apoptotic Cell Death in Malignant Tumor Cells and Natural Compounds. Cancer Lett 2018;420:210-27.

14. Gu C, Yin Z, Nie H, et al. Compound kushen injection relieves tumor-associated macrophage-mediated immunosuppression through TNFR1 and sensitizes hepatocellular carcinoma to sorafenib. BMC Biol 2020;18:1-17.

15. Chang FL, Tsai KC, Lin TY, et al. Astragalus membranaceus-Derived Anti-Programmed Death-1 Monoclonal Antibodies with Immunomodulatory Therapeutic Effects against Tumors. Biomed Res Int 2020;2020:3415471.

16. Luo H, Vong CT, Chen H, et al. Naturally occurring anticancer compounds: shining from Chinese herbal medicine. Chin Med 2019;14:48.

17. Tian G, Wu C, Li J, et al. Network Pharmacology Based investigation into the Effect and mechanism of Modified Sijunzi Decoction against the subtypes of Chronic Atrophic Gastritis. Pharmacol Res 2019;144:158-66.

18. Committee NP. Pharmacopoeia of the People's Republic of China. Beijing: Chinese medicine Science and Technology Press, 2010.

19. Ye Q, Su L, Chen D, et al. Astragaloside IV Induced miR-134 Expression Reduces EMT and Increases Chemotherapeutic Sensitivity by Suppressing CREB1 Signaling in Colorectal Cancer Cell Line SW-480. Cell Physiol Biochem 2017;43:1617-26.

20. Liu Y, Liu J, Wu KX, et al. A rapid method for sensitive profiling of bioactive triterpene and flavonoid from,

Cite this article as: Wang Q, Dou Y, Zhao S, Wei M, Sun D, He Y, Zhu D, Chen X, Zhu H. Analysis of chemical consistency and the anti-tumor activity of Huangqi-Ezhu (HQEZ) concentrated-granules and decoction. Ann Palliat Med 2020;9(4):1648-1659. doi: 10.21037/apm-19-592
Astragalus mongholicus, and, Astragalus membranaceus, by ultra-pressure liquid chromatography with tandem mass spectrometry. J Chromatogr B Analyt Technol Biomed Life Sci 2018;1085:110-8.

21. Wu J, Wang J, Su Q, et al. Traditional Chinese medicine Astragalus polysaccharide enhanced antitumor effects of the angiogenesis inhibitor apatinib in pancreatic cancer cells on proliferation, invasiveness, and apoptosis. Onco Targets Ther 2018;11:2685-98.

22. Valdivieso-Ugarte M, Gomez-Llorente C, Plaza-Díaz J, et al. Antimicrobial, Antioxidant, and Immunomodulatory Properties of Essential Oils: A Systematic Review. Nutrients 2019;11:2786.

23. García-Quiroz J, García-Becerra R, Santos-Cuevas C, et al. Synergistic Antitumorigenic Activity of Calcitriol with Curcumin or Resveratrol is Mediated by Angiogenesis Inhibition in Triple Negative Breast Cancer Xenografts. Cancers (Basel) 2019;11:1739.

24. Deng M, Liu B, Song H, et al. $\beta$-Elemene inhibits the metastasis of multidrug-resistant gastric cancer cells through miR-1323/Cbl-b/EGFR pathway. Phytomedicine 2020;69:153184.

25. Wei W, Rasul A, Sadiqa A, et al. Curcumol: From Plant Roots to Cancer Roots. Int J Biol Sci 2019;15:1600-9.

26. Min FU, Yalin S, Zengtao S. Comparative Study on Modern Application of Traditional Chinese Medicine Decoction and Traditional Chinese Medicine Granule Preparation. Chinese Medicine Modern Distance Education of China 2017;15:152-4. 